\title{
Surface plasmon-polariton study of the optical dielectric function of titanium nitride
}

\author{
A. P. HIBBINS and J. R. SAMBLES
}

Thin Film Photonics Group, Department of Physics, University of Exeter, Exeter EX4 4QL, England

and

\section{R. LAWRENCE}

Defence Evaluation and Research Agency, Farnborough GU14 OLX, England.

\begin{abstract}
This work presents the first detailed study of the optical dielectric function of optically thick $\operatorname{TiN}_{x}$ films using grating-coupling of radiation to surface plasmon-polaritons. Angle-dependent reflectivities are obtained in the wavelength range $500-875 \mathrm{~nm}$ and by comparison with grating modelling theory, we determine both the imaginary and real parts of the dielectric function. This method provides an alternative to traditional characterisation techniques (e.g. Kramers-Kronig analysis) that may require additional information about film thickness, or the sample's optical properties in other parts of the electromagnetic spectrum. We
\end{abstract}


have fitted the determined dielectric function to a model based on a combination of interband absorptions and free-electron response evaluating both the plasma energy and relaxation time.

\section{Introduction}

Titanium nitride $\left(\mathrm{TiN}_{\mathrm{x}}\right)$ belongs to a group of transition metal compounds that are of considerable interest because they have a variety of properties which lend themselves to many potential applications. For example, $\mathrm{TiN}_{x}$ has become widely used as a wearresistant coating on tools because of its mechanical resistance, low friction coefficient and high melting point [1]. Its high chemical stability and low resistivity also make it a good candidate as a diffusion barrier in semiconductor metallization systems [2,3]. $\mathrm{TiN}_{x}$ is also fascinating in that it appears gold in colour owing to the displacement of the plasma edge into the visible range by the onset of interband transitions [4,5]. Hence it is often used as a scratch-resistant alternative to gold on decorative jewellery. Its high Drude-like reflectance in the infra-red and high absorptivity in the visible and near infra-red have encouraged its use as a selective absorber and heat mirror. Unfortunately the plasma edge is located at too high an energy to make it an efficient solar absorber in its stoichiometric form $[4,6,7]$. However it is well known that the plasma energy and the optical and electrical properties of $\operatorname{TiN}_{x}$ films are strongly dependent on the deposition conditions, film thickness and titanium-nitrogen ratio [6-12]. This 
present study is concerned with exploring these interesting optical characteristics in the visible part of the spectrum using the optical excitation of surface plasmons.

To our knowledge, the first multi-wavelength study of the optical constants of $\operatorname{TiN}_{x}$ was carried out by Böhm et al. [8] in 1972. They calculated the optical dispersion and absorption parameters of $\mathrm{Ti}(\mathrm{C}, \mathrm{N})$ solid solutions by means of ellipsometric measurements between energies of 0.6 and $5.5 \mathrm{eV}$. Their results illustrate the Drude-like response of $\mathrm{TiN}_{0.94}$ at low energies and its disturbance by the onset of interband transitions as the photon energy is increased. Schlegel et al. [5] characterised $\mathrm{TiN}_{x}$ samples using Kramers-Kronig analysis [13] of reflectivity measurements carried out between 0.3 and $12 \mathrm{eV}$. Samples were prepared by chemical vapour deposition (CVD) on to heated crystals of $\mathrm{TiN}_{x}$. Karlsson et al. [4] and Rivory et al. [6] both obtained the dielectric function of opaque (thickness, $d>100 \mathrm{~nm}$ ), stoichiometric $\operatorname{TiN}_{x}$ samples prepared by reactive sputtering on to fused-silica and glass substrates respectively. Kramers-Kronig analysis was again used to determine the optical permittivities between 0.02 and $6.2 \mathrm{eV}$ (Karlsson) and 0.5 and 6 eV (Rivory). Szczyrbowski et al. [10] and Valkonen et al. [12] determined the optical constants of sputtered $\mathrm{TiN}_{x}$ films in the visible and near infrared spectral regions from measurements of transmission, reflectance and film thickness $(\mathrm{d}<$ $31 \mathrm{~nm})$. Pascual et al. [11] deposited opaque $\mathrm{TiN}_{x}$ films by plasma- 
assisted chemical vapour deposition (PACVD) and determined its dielectric function by means of spectroscopic ellipsometry in the UV-visible range.

The use of grating-coupled surface plasmon-polaritons (SPP) [14] in the study of the profile and permittivity of metal surfaces provides an alternative method to the techniques described above. The SPP comprises of a surface charge density oscillation and associated electromagnetic fields, propagating along a metal/dielectric interface. Owing to the reversal of the normal component of the electric field at the surface, the SPP is very strongly localised in the plane, and so is extremely sensitive to the dielectric properties of the media either side of the interface. However, on a flat surface, the wave vector of the SPP is greater than the maximum photon wave vector available in the adjacent dielectric and therefore it can neither be directly coupled to, nor can it radiate. The enhanced wave vector required to couple into the mode may be provided by either increasing the wave vector of the incident radiation by using a high index prism $[15,16]$ or a corrugated interface. The latter is usually in the form of an optical diffraction grating, where the in-plane wave vector enhancement is in multiples of the grating wave vector $\boldsymbol{k}_{g}$ [17]. By varying the magnitude of the incident wave vector in the plane of the grating, wave vector values are found at which incident radiation may 
couple resonantly to the SPP mode. Experimentally, the excitation of a SPP is observed as a dip in the angle-dependent reflectivity.

Previously, the grating-coupled SPP method has successfully been used to characterise metals including copper [18] and silver [19], and Steinmüller-Nethl et al. [20] have reported the excitation of SPPs on a thin $\operatorname{TiN}_{x}$ film sputtered onto a sinusoidally modulated substrate. They recorded angle-dependent reflectivities at four incident wavelengths in the visible regime and compared them with those predicted from a rather simplistic treatment of the interaction of radiation with the surface. This allowed them to estimate the dielectric function of $\operatorname{TiN}_{x}$ at these wavelengths. The grating-coupled SPP method presents many advantages over the other techniques described previously. For example, it does not require transmittivity data or the determination of film thickness, hence permitting the use of optically opaque metallic films. It can also be used to calculate the permittivity of a material over a limited wavelength range without the knowledge of its optical response elsewhere in the electromagnetic spectrum - there is no Kramers-Kronig based interpretation.

This present study uses grating-coupling of radiation to SPPs on a $\operatorname{TiN}_{x}$ surface to obtain the dispersion relation of the $\mathrm{TiN}_{x}$ film. Values of $\varepsilon$ are determined at visible wavelengths by comparison of the in-plane reflectance from the corrugated $\mathrm{TiN}_{x}$-air interface as a 
function of the angle of incidence, to the predictions from a rigorous grating theory model which uses the differential formalism of Chandezon et al. [21]. By varying the grating parameters used to generate a theoretical reflectivity trace, it is possible to obtain a good quality fit to the experimental data and hence determine the shape [22] and optical permittivity $[18,19]$ of the $\operatorname{TiN}_{x}$ surface.

\section{Experimental}

The corrugated surface used in this work was prepared in silica by standard interferographic techniques [23]. The method of preparation involves the exposure of spin-coated photoresist on a silica disc to the interference pattern produced by first splitting and then recombining an argon-ion laser beam. After exposure, the photoresist is chemically developed and the resulting profile transferred into the silica by fast atom etching. This produces a robust and large area diffraction grating with a well-defined pitch (in this case $\lambda_{g}=930 \mathrm{~nm}$ ) and a uniform surface profile that is somewhat distorted from a pure sinusoid. Subsequent deposition of an optically thick ( $d \approx 600 \mathrm{~nm}$ ) layer of titanium nitride produces a metallic-air interface that may support SPPs. 
The $\operatorname{TiN}_{x}$ film was deposited by TEER Coatings Ltd (Kidderminster, UK) using the closed field unbalanced magnetron sputter ion plating system [24]. The substrate is ion-cleaned before deposition which ensures a high degree of adherence to the silica surface. Initial sputtering is from a pure titanium target which is "poisoned" by bleeding nitrogen into the coating chamber. In order to attempt to control the titanium/nitrogen ratio of the sputtered sample, the intensity of $501 \mathrm{~nm}$ radiation emitted from the target is monitored. From past experience, it is thought that approximately stoichiometric $\operatorname{TiN}_{x}$ may be deposited on the substrate when the intensity of radiation falls to $60 \%$ of the original value. The coating pressure in the chamber, back filled with Ar gas, was $4 \times 10^{-3}$ Torr and the substrate bias voltage and temperature were $30 \mathrm{~V}$ and approximately $150{ }^{\circ} \mathrm{C}$ respectively.

Previous workers, for example [11], have shown the real part of the dielectric function of $\operatorname{TiN}_{x}$ to be negative, and therefore able to support interface modes, at wavelengths in excess of approximately $500 \mathrm{~nm}$. The wave vector of the incident radiation in the dielectric is $n_{d} \underline{k}_{0}$ (where $k_{0}=\omega / c=2 \pi / \lambda_{0}$ and $n_{d}=1.0003$ is the refractive index of air) and we may only couple to the SPP when the in-plane component of this has been suitably enhanced. This mechanism is provided by the grating's periodicity, which may increase or decrease the in-plane component of $n_{d} \underline{k}_{0}$ by integer multiples of 
the grating wave vector, $\underline{k}_{g}\left(k_{g}=2 \pi / \lambda_{y}\right)$. This gives rise to diffracted orders which no longer propagate in the dielectric when their in-plane wave vector is increased such that it becomes greater than that of the incident radiation. It is the enhanced wave vector of these evanescent fields that allows incident radiation to couple to the SPP according to the condition

$$
\underline{k}_{S P P}=\left(n_{d} k_{0} \sin \theta\right) \underline{\hat{x}} \pm N \underline{k}_{g}
$$

(1).

Here $N$ is an integer, $\underline{k}_{S P P}$ is the wave vector of the SPP and $n_{d} k_{0} \sin \theta$ is the projection of the wave vector of the incident radiation parallel to $\boldsymbol{k}_{g}$ (along the $x$-axis). If the angle between the plane of incidence and the grating vector (called the azimuthal angle, $\varphi$ ) is equal to zero then all the diffracted beams lie in the plane of measurement and equation 1 reduces to a scalar equation.

The SPP resonances were monitored by recording the specularly reflected intensity from the $\mathrm{TiN}_{x}$-air interface as a function of the angle of incidence (figure 1). Monochromatic, p-polarised (transverse magnetic) radiation, of wavelength ranging from 500 to $875 \mathrm{~nm}$ in $25 \mathrm{~nm}$ steps, was incident in a plane parallel to the grating wave vector $\left(\boldsymbol{\varphi}=0^{\circ}\right)$. The normalised experimental angledependent reflectivity data was fitted by a least-squares procedure using a scattering matrix [25] technique based on the differential formalism of Chandezon et al. [21]. This theory involves 
transforming the mathematics into a new frame in which the boundary conditions at the corrugated surface are more readily solved - effectively flattening the surface. This requires that the incident field is represented as a Fourier expansion. The surface itself is also described by a Fourier series $A(x)=a_{1} \cos \left(k_{g} x\right)+a_{2} \cos \left(2 k_{g} x\right)+. .+a_{N} \cos \left(N k_{g} x\right)+.$.

(2).

By fitting the reflectivity data to the modelling theory, it is possible to parameterise the grating profile and characterise the dielectric function of the metallic material.

In order to provide an accurate description of the complex dielectric function of $\operatorname{TiN}_{x}$, it is first necessary to be confident of the surface profile. In the visible regime, it is well documented [for example, 19] that the real part of the dielectric function of silver is more negative, and the imaginary part much smaller than the corresponding values for $\operatorname{TiN}_{x}$. Hence the surface plasmon resonances on a silver/air interface are far better defined, consequently giving more sensitivity to the grating profile. Therefore, in order to obtain a good first approximation to the profile of the $\mathrm{TiN}_{x}$-coated surface, we thermally evaporate an optically thick layer of silver on top of the $\operatorname{TiN}_{x}$. The pitch of the grating was chosen so that when $\varphi=0^{\circ}$, incident light of wavelength, $\lambda_{0}=590 \mathrm{~nm}$ will excite first, second and third order 
SPP resonances $(N=1,2,3)$ which may be observed as resonant features in an angle of incidence scan. By fitting the optical data from the silver surface to the predicted optical response using the theory described above, we are able to provide a good estimate of the three Fourier amplitudes of the corrugated surface that provide the direct coupling mechanism for the three SPP resonances. This truncated Fourier series can then be used in the fitting of data obtained from the $\mathrm{TiN}_{x}$-coated surface over the whole spectral regime. Subsequently, after further fitting, allowing all the surface parameters to vary, we obtain a new profile for the $\operatorname{TiN}_{x}$ surface by averaging the Fourier components over all wavelengths. This new average profile is then held constant at all wavelengths and the data finally refitted in order to obtain the complex dielectric constant at each of the wavelengths studied.

\section{Results}

Figure 2 shows two sets of typical reflectivity data at incident wavelengths of $650 \mathrm{~nm}$ and $800 \mathrm{~nm}$. The solid lines are the theoretical fits, which are in very good agreement with the data (circles). The optical properties of $\mathrm{TiN}_{x}$ are often likened to those of gold, but the off-resonance reflectivity of less than $50 \%$ is clearly less than the $\sim 90 \%$ to be expected from gold [26]. In addition, the resonances themselves are broad and shallow. This indicates that 
$\mathrm{TiN}_{x}$, compared to gold, has an increased $\mathcal{\xi}_{\boldsymbol{i}}$ and probably a less negative $\boldsymbol{\varepsilon}_{\boldsymbol{r}}$.

As indicated above, the profile of the grating is initially estimated from the optical characterisation of the silver surface, and then improved by fitting to the $\operatorname{TiN}_{x}$ data. The pitch, as defined by the angular positions of the pseudo-critical edges in the experimental reflectivity data was calculated to be $\lambda_{g}=930.1 \pm 0.5 \mathrm{~nm}$ with the permittivity of the dielectric (air) set as $\varepsilon_{d}=n_{d}^{2}=1.0006$. The values of the complex dielectric constants of the $\mathrm{TiN}_{x}$ found from the final fits to the reflectivity data are listed in table 1 $\left(\varepsilon_{\mathrm{TiN}}=\varepsilon_{r}+i \varepsilon_{i}\right)$. The errors in these values are dominated by an uncertainty in the Fourier coefficients of the grating profile. This is attributed to the low sensitivity of the broad and shallow resonance features to the various Fourier coefficients. There is also a variation in the uncertainty over the wavelength range due to a change in the number of SPP resonances observable at different wavelengths in the angle of incidence scans. In addition, there is an increase in the uncertainty when two reflectivity minima overlap, and also as the coupling strength to the resonances decrease as we approach the limit $\varepsilon_{r}=0$. The results presented in this work, along with those of Karlsson et al. [4], Rivory et al. [6], 
Szczyrbowski et al. [10], Pascual et al. [11], Valkonen et al. [12] and Steinmüller-Nethl et al. [20] are illustrated in figure 3.

\section{Discussion}

It is well known that the optical properties of $\mathrm{TiN}_{x}$ films are strongly dependent on the deposition conditions, film thickness and the titanium/nitrogen ratio. Therefore, the variation in the values of $\boldsymbol{\varepsilon}_{\boldsymbol{r}}$ and $\boldsymbol{\varepsilon}$, as illustrated in figure 3 , is not surprising. The works of Karlsson et al. [4] and Rivory et al. [6] both consider opaque samples $(d>100 \mathrm{~nm})$ of similar stoichiometries $(x=1)$, and use identical characterisation techniques in order to obtain the optical constants. As a result, the dielectric function of their respective samples compare favourably. Szczyrbowski et al. [10] and Valkonen et al. [12] have both sputtered much thinner films ( $d<31 \mathrm{~nm}$ ) and although their values of $\mathcal{F}_{r}$ are comparable to those of other workers, their values of $\mathcal{\varepsilon}_{i}$ are considerably higher due to increased electron scattering. The "thin" films obtained by PACVD and investigated over visible wavelengths by Pascual et al. [11] show the most anomalous results. It is not clear why their results give values for both parts of the complex dielectric function which are smaller in magnitude than other studies. SteinmüllerNethl et al. [20] used a grating-coupled SPP technique to characterise the optical properties of their magnetron-sputtered film at four wavelengths. They appear to have been the first to 
record SPP excitation on $\mathrm{TiN}_{x}$. Their films were of comparable thickness to those of Szczyrbowski et al. [10] and Valkonen et al. [12] $(d=50 \mathrm{~nm})$, however they do not show the associated increase in $\boldsymbol{\varepsilon}$. However, it should be noted that their data fitting technique is based on a pertubative approach to Fresnel equations, and they assume shallow (depth/pitch < 0.07), purely sinusoidal gratings. In view of the fact that their grating is produced by interferographic exposure, followed by ion-etching, then the profile is likely to be distorted significantly from the assumed shape. This results in poor quality fits and the errors associated with their deduced values of $\boldsymbol{\varepsilon}_{\boldsymbol{r}}$ and $\boldsymbol{\varepsilon}$ will be large.

In this work we present a comprehensive study of the SPP on $\operatorname{TiN}_{x}$ using a rigorous model of the optical response of metallic gratings to fit the experimental reflectivity data from an optically thick $(d \approx 600 \mathrm{~nm})$ sputtered film. The grating profile is accurately modelled as a distorted sinusoidal surface by representing it as a truncated Fourier series and using the theoretical model described previously. From this we produce fits that show excellent agreement with the experimental reflectivities (figure 2). The experimentally determined dielectric function (figure 3) illustrates that $\boldsymbol{\varepsilon}_{\boldsymbol{r}}$ is slightly smaller in magnitude than deduced by other workers for similar films $[4,6]$, however $\mathcal{\xi}$ shows good agreement with these other studies. 
If the dielectric function of $\mathrm{TiN}_{x}$ is dominated by a free-electron behaviour at the wavelengths studied in this work, we would expect our data to conform to the Drude model, i.e.

$$
\varepsilon_{r}(\omega)=\varepsilon_{0}-\frac{\omega_{p}^{2} \tau^{2}}{1+\omega^{2} \tau^{2}}
$$

$$
\varepsilon_{i}(\omega)=\frac{\omega_{p}^{2} \tau}{\omega\left(1+\omega^{2} \tau^{2}\right)}
$$

\section{(4)}

where $\tau$ is the relaxation time, $\omega_{p}$ is the plasma frequency and $\varepsilon_{0}$ is residual dielectric constant (conventionally assumed to be unity). Therefore we should be able to plot $\mathcal{E}_{r}$ against $\mathcal{X}$ and $\mathcal{\varepsilon}_{\boldsymbol{i}}$ against $\not{X}$ and expect linear relationships. Plotting our data in this way gives the curves of figure 4 (a) and (b) respectively. Figure 4 (a) shows a high degree of linearity, the $y$-intercept indicating a residual dielectric constant of approximately +3 . This can be attributed to a positive contribution to $\mathcal{E}_{\mathrm{r}}$ from the occurrence of interband transitions as predicted by a number of previous workers [4-8]. The linear fit to $\mathcal{\xi}$ is less convincing and hence also suggests that the optical response of $\mathrm{TiN}_{x}$ is not purely Drude-like in nature. In addition, previous workers have illustrated that the point at which $\varepsilon_{r}=0$ is situated at a much lower energy than the 
predicted plasma resonance. The crossing of the $\varepsilon_{r}=0$ axis is associated with a screened plasmon mode which has been shifted to lower energies by the onset of interband transitions at photon energies above $2.5 \mathrm{eV}$ [4]. Consequently, any treatment of the complex dielectric constant close to this region must involve splitting it into two parts, one part corresponding to intraband excitations described by the Drude model, and an interband part corresponding to resonant absorptions based on a Lorentz oscillator model, i.e.

$$
\varepsilon_{r}(\omega)=\varepsilon_{r}^{D}(\omega)+\varepsilon_{r}^{I}(\omega)
$$

\section{(5)}

and

$$
\varepsilon_{i}(\omega)=\varepsilon_{i}^{D}(\omega)+\varepsilon_{i}^{I}(\omega)
$$

(6).

We fit our data to a model that assumes only one resonant interband transition. This is a reasonable assumption to make since we expect peaks in $\varepsilon_{i}(\omega)$ corresponding to strong interband transitions at approximately $3.7 \mathrm{eV}$ and $5.2 \mathrm{eV}[4,6,27]$, but have only determined the dielectric function at energies up to $2.5 \mathrm{eV}$. Hence we will only experience the effects of the wing of the lower energy resonance. The two parts of the complex dielectric function are therefore 


$$
\varepsilon_{r}(\omega)=\varepsilon_{0}-\frac{\omega_{p}^{2} \tau^{2}}{1+\omega^{2} \tau^{2}}+\frac{\tau^{2} \omega_{p}^{2}\left(\omega_{0}^{2}-\omega^{2}\right)}{\tau^{2}\left(\omega_{0}^{2}-\omega^{2}\right)^{2}+\omega^{2}}
$$

and

$$
\varepsilon_{i}(\omega)=\frac{\omega_{p}^{2} \tau}{\omega\left(1+\omega^{2} \tau^{2}\right)}+\frac{\omega_{p}^{2} \omega \tau}{\tau^{2}\left(\omega_{0}^{2}-\omega^{2}\right)^{2}+\omega^{2}}
$$

(8).

By rearranging equations 7 and 8 and putting $\varepsilon_{0}=1$, we eliminate cos such that

$$
\frac{\varepsilon_{r}(\omega)-1}{\varepsilon_{i}(\omega)}=\omega \tau\left[\left(\frac{\omega_{0}^{2}\left(\omega^{2} \tau^{2}+1\right)}{\omega^{2}\left(\omega^{2} \tau^{2}+1\right)+\tau^{2}\left(\omega_{0}^{2}-\omega^{2}\right)^{2}+\omega^{2}}\right)-1\right]
$$

(9).

From this it is possible to obtain the constants $\omega_{0}=3.9 \mathrm{eV}$ and $/ \tau=\mathbf{1 1} \mathrm{eV}$ by fitting the functional form of equation 9 to experimentally derived data (figure 5). The fit to the experimental data illustrated in figure 5 is rather poor, but it is important to note that reflectivity measurements were carried out over a very limited frequency range. However the general upward trend in the experimental data is apparent in the model, and the value of compares favourably with the energy of the first peak in $\boldsymbol{\varepsilon}_{i}(\boldsymbol{\omega})$ determined by previous workers $(3.8 \pm 0.1 \mathrm{eV}[4,6,27])$. We may then fit to capy using equations 7 and 8 (figure 6 a and b). A 
comparison of our values of $\boldsymbol{c}_{\boldsymbol{\beta}}$ and $\tau$ to those of previous workers is shown in table 2 .

On examination of table 2 , it is apparent that there is a large spread of values of both cos and $\tau$, dependent on the deposition conditions and stoichiometry of the sample. The plasma frequency and relaxation time calculated in the present study are among the lowest observed, indicating a sample of reduced metallic nature which can at least in part be attributed to a large nitrogen/titanium ratio $(x)$. Indeed, this is confirmed by electron energy dispersive spectroscopy measurements which indicate that $x$ is well in excess of unity.

\section{Conclusions}

In this paper, for the first time, we have determined the dielectric function of $\operatorname{TiN}_{x}$ throughout the visible regime using a gratingcoupled SPP resonance technique. This technique presents a number of advantages over more conventional characterisation methods. For example, we need only take reflectivity data at the individual wavelengths at which we wish to calculate the dielectric function, and we require no measurement of the film thickness. We have illustrated that we are able to obtain excellent agreement between the differential formalism of Chandezon and experimental 
reflectivities, and the resulting dielectric function compares favourably with that determined by previous workers. By making the assumption that $\operatorname{TiN}_{x}$ is only free-electron like in its optical response, we have shown that the Drude model alone is too simplistic. Instead, we have fitted the experimentally determined dielectric function to a Drude-Lorentz model that assumes a single resonant interband transition, in order to obtain the plasma frequency and relaxation time. A comparison of constants determined from this work and previous studies shows a large spread of results, illustrating the dependence on the stoichiometry and deposition conditions of the sample.

\section{Acknowlegements}

The authors would like to acknowledge the financial support of the Defence Evaluation and Research Agency, Farnborough, and wish to thank Jeffery Jones (Earth Resources Centre, University of Exeter) for his contribution to the EDS study.

\section{References}

[1] Munz, W. D., Hofmann, D. and Hartig, K., 1986, Thin Sold Films, 96, 79 .

[2] Kanamori, S., 1986, Thin Solid Films, 136, 195. 
[3] Gagnon, G., Currie, J. F., Brebner, J. L., and Darwell, T., 1996, J. Appl. Phys., 79, 7612.

[4] Karlsson, B., Sundgren, J.-E, and Johansson, B.-O, 1982, Thin Solid Films., 87, 181.

[5] Schlegel, A., Wachter, P., Nickl, J. J., and Lingg, H., 1977, J. Phys.

$C, \mathbf{1 0}, 4889$.

[6] Rivory, J., Behaghel, J. M., Berthier, S., and Lafait, J., 1981, Thin Solid Films, 78, 161.

[7] François, J. C., Chassaing, G., Gravier, P., Pierrisnard, R., and Bonnot, A. M., 1985, Thin Solid Films, 127, 205.

[8] Böнm, G., and Goretzki, H. 1972, J. Less-Common Metals, 27, 311.

[9] Kurtz, S. R., and Gordon, R. G., 1986, Thin Solid Films, 140, 277.

[10] Szczyrbowski, Von J., Rögels, S., Kastner, A., Dietrich, A., and Hartig, K., 1988, Vakuum-Technik, 37, 14.

[11] Pascual, E., Polo, M. C., Esteve, J., and Bertran, E., 1991, Surface Science, 251-252, 200.

[12] Valkonen, E., Ribbing, C.-G., and Sundgren, J.-E., 1986, Proc. SPIE, 652, 235.

[13] Wooten, F., 1972, Optical Properties of Solids (New York: Academic Press), chapter 6.

[14] Raether, H., 1988, Surface Plasmons on Smooth and Rough Surfaces and on Gratings, (Berlin: Springer).

[15] Kretschmann E., and Raether, H., 1968, Z. Naturf., 23a, 2135. 
[16] Отто, А., 1968, Z. Phys., 216, 398.

[17] Teng, Y.-Y., and Stern, E. A., 1967, Phys. Rev. Lett., 19, 511.

[18] Nash, D. J., and Sambles, J. R., 1995, J. Mod. Opt., 42, 1639.

[19] Nash, D. J., and Sambles, J. R., 1996, J. Mod. Opt., 43, 81.

[20] Steinmüller-Nethl, D., Kovacs, R., Gornik, E., and Rödhammer, P., 1994, Thin Solid Films, 237, 277.

[21] Chandezon, J., Dupuis, M. T., Cornet, G., and Maystre, D., 1982, J. Opt. Soc. Am., 72, 839.

[22] Wood, E. L., Sambles, J. R., Cotter, N. P., and Kitson S. C., 1995, J. Mod. Opt, 42, 1343.

[23] Hutley, M. C., 1982, Diffraction Gratings (London: Academic Press).

[24] Monaghan, D. P., Teer, D. G., Laing, K. C., Efeoglu, I., and Arnell, R. D., 1993, Surf. and Coatings Technol., 59, 21.

[25] Cotter, N. P. K., Priest, T. W., and Sambles, J. R., 1995, J. Opt. Soc. Am., 12, 1097.

[26] Watts, R. A., Sambles, J. R., and Harris, J. B., 1997, Optics Commun., 135, 189.

[27] Karlsson, B., Shimshock, R. P., Seraphin, B. O., and Haygarth, J. C., 1983, Sol. Energy Mater., 7, 401.

\section{Table Captions}


Table 1. The optical dielectric function of titanium nitride experimentally determined in this work, together with estimated errors.

Table 2. A comparison of the values of $\omega_{p}$ and $/ \tau$ of $\operatorname{TiN}_{x}$ experimentally determined in this work, along with the values obtained by a number of previous workers. The nitrogen/titanium ratio $(x)$ and deposition method is also listed for comparison. 


\section{Figure Captions}

Figure 1. Schematic representation of the experimental set up used to record angle dependent reflectivity data from a diffraction grating. In order for the signal detector to track the specularly reflected beam, the signal detector rotates at twice the rate of the grating mounted on the table.

Figure 2. The experimental angle-dependent reflectivities $(O$, one point in five plotted for clarity) compared with the theoreticallymodelled reflectivities (-) created from a single set of parameters describing the grating profile. (a) and (b) show $R_{p p}$ reflectivity measurements (i.e. p-polarised (TM) radiation was incident and detected) at incident wavelengths of $650 \mathrm{~nm}$ and $800 \mathrm{~nm}$ respectively. The values of the dielectric constants at these wavelengths that were obtained by fitting the model to the experimental data are shown in table 1.

Figure 3. A comparison of the values of the dielectric function of $\mathrm{TiN}_{x}$ determined in this work (O), with the results of Karlsson et al. [4] ( ), Rivory et al. [6] ( ), Szczyrbowski et al. [10] (- - -), Pascual et al. [11] ( ), Valkonen et al. [12] ( ) and Steinmüller-Nethl et al. [20] (一). 
Figure 4. A plot of (a) $\mathcal{\varepsilon}_{r}$ against $\lambda_{\theta}^{2}$ and (b) $\mathcal{\varepsilon}_{i}$ against $\lambda_{g}^{3}$ for the data presented in this work. A high degree of linearity suggests that the $\operatorname{TiN}_{x}$ sample is free-electron like. However the $y$ intercept of (a) is greater than $\varepsilon_{0}=1$, attributable to a positive contribution to $\boldsymbol{\varepsilon}_{\boldsymbol{r}}$ from the occurrence of interband transitions. The errors illustrated in the values of the dielectric constants are dominated by an uncertainty in the grating profile.

Figure 5. By assuming a one-resonance Drude-Lorentz model, we obtain a function relating the two parts of the complex dielectric function, dependent only on $\omega, \boldsymbol{\omega}$ and $\tau$ (equation 9). Values of this function calculated using the experimentally determined dielectric constants and uncertainties (error bars) are compared with the fitted theoretical function (solid line). We are thus able to determine the values of $\boldsymbol{\xi}$ and $\tau$.

Figure 6. The (a) real part, (b) imaginary part, and the associated uncertainties in the dielectric function of $\mathrm{TiN}_{x}$ determined in the present work. We have modelled the experimental data (error bars) using equations 7 and 8 in order to obtain the plasma frequency, cop. The resulting fit is shown as a solid line. 


\begin{tabular}{|c|c|c|c|c|c|c|c|c|c|}
\hline $\begin{array}{c}\lambda_{\mathbf{0}} \\
(\mathbf{n m} \\
)\end{array}$ & $\varepsilon$ & $\delta E_{1}$ & $\varepsilon_{i}$ & $\varepsilon_{1}$ & $\begin{array}{c}\lambda_{0} \\
(\mathbf{n m} \\
)\end{array}$ & $\varepsilon$ & $E_{\varepsilon_{r}}$ & $\varepsilon$ & $\varepsilon_{i}$ \\
\hline 500 & -0.20 & $\begin{array}{c} \pm \\
0.05\end{array}$ & 2.41 & $\begin{array}{c} \pm \\
0.15\end{array}$ & 700 & -3.58 & $\begin{array}{c} \pm \\
0.10\end{array}$ & 5.36 & $\begin{array}{c} \pm \\
0.10\end{array}$ \\
\hline 525 & -0.64 & 0.10 & 3.12 & 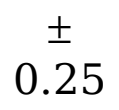 & 725 & -4.23 & $0 . \stackrel{ \pm}{10}$ & 5.74 & 0.20 \\
\hline 550 & -1.07 & $\begin{array}{c} \pm \\
0.10\end{array}$ & 3.37 & $\begin{array}{c} \pm \\
0.10\end{array}$ & 750 & -4.84 & $\begin{array}{c} \pm \\
0.10\end{array}$ & 6.07 & 0.20 \\
\hline 575 & -1.43 & $\begin{array}{c} \pm \\
0.10\end{array}$ & 3.79 & $\begin{array}{c} \pm \\
0.10\end{array}$ & 775 & -5.19 & $\begin{array}{c} \pm \\
0.05\end{array}$ & 6.24 & $\begin{array}{c} \pm \\
0.20\end{array}$ \\
\hline 600 & -1.91 & 0.10 & 4.17 & 0.10 & 800 & -5.89 & $\begin{array}{c} \pm \\
0.05\end{array}$ & 6.64 & 0.20 \\
\hline 625 & -2.35 & $\begin{array}{l} \pm \\
0.05\end{array}$ & 4.30 & $\begin{array}{c} \pm \\
0.30\end{array}$ & 825 & -6.03 & $\stackrel{ \pm}{ \pm}$ & 6.96 & 0.25 \\
\hline $\begin{array}{c}632 . \\
8\end{array}$ & -2.61 & $\begin{array}{c} \pm \\
0.05\end{array}$ & 4.36 & $\begin{array}{c} \pm \\
0.30\end{array}$ & 850 & -6.67 & $\stackrel{ \pm}{ \pm}$ & 7.60 & $\stackrel{ \pm}{ \pm}$ \\
\hline 650 & -2.88 & $\begin{array}{c} \pm \\
0.25\end{array}$ & 4.75 & $\begin{array}{c} \pm \\
0.30\end{array}$ & 875 & -7.51 & $0 . \stackrel{ \pm}{20}$ & 8.15 & 0.30 \\
\hline 675 & -3.02 & $\begin{array}{c} \pm \\
0.15\end{array}$ & 5.18 & $\begin{array}{c} \pm \\
0.10\end{array}$ & & & & & \\
\hline
\end{tabular}




\begin{tabular}{|c|c|c|c|}
\hline $\begin{array}{c}\text { Sample } \\
\text { [reference] } \\
\text { (preparation } \\
\text { method) }\end{array}$ & $x$ & $\begin{array}{c}\omega_{\mathrm{p}} \\
(\mathrm{eV})\end{array}$ & $\begin{array}{c}/ \tau \\
(\mathbf{e V})\end{array}$ \\
\hline $\begin{array}{l}\text { Karlsson } \\
\text { [4] (CVD) } \\
\text { Schlegel }\end{array}$ & 1 & 6.29 & 0.30 \\
\hline $\begin{array}{l}\text { [5] (CVD) } \\
\text { Rivory }\end{array}$ & - & $6.9-7.0$ & $0.6-0.8$ \\
\hline $\begin{array}{l}\text { (reactive } \\
\text { sputtering) } \\
\text { Böhm }\end{array}$ & $\approx 1$ & 8.7 & 1.3 \\
\hline $\begin{array}{l}\text { [8] } \\
\text { solution) } \\
\text { Kurtz }\end{array}$ & 0.94 & 8.9 & 1.22 \\
\hline $\begin{array}{l}{[9]} \\
\text { (CVD) } \\
\text { SzCZyrbowski }\end{array}$ & - & $5.62-6.25$ & $0.93-1.27$ \\
\hline $\begin{array}{l}\text { [10] (reactive } \\
\text { sputtering) } \\
\text { Valkonen }\end{array}$ & 1.16 & 7.5 & 1.1 \\
\hline [12] (reactive & - & $7.0-7.1$ & $0.4-0.6$ \\
\hline $\begin{array}{l}\text { sputtering) } \\
\text { Present Data } \\
\text { (reactive } \\
\text { sputtering) }\end{array}$ & $>1$ & 5.8 & 1.1 \\
\hline
\end{tabular}




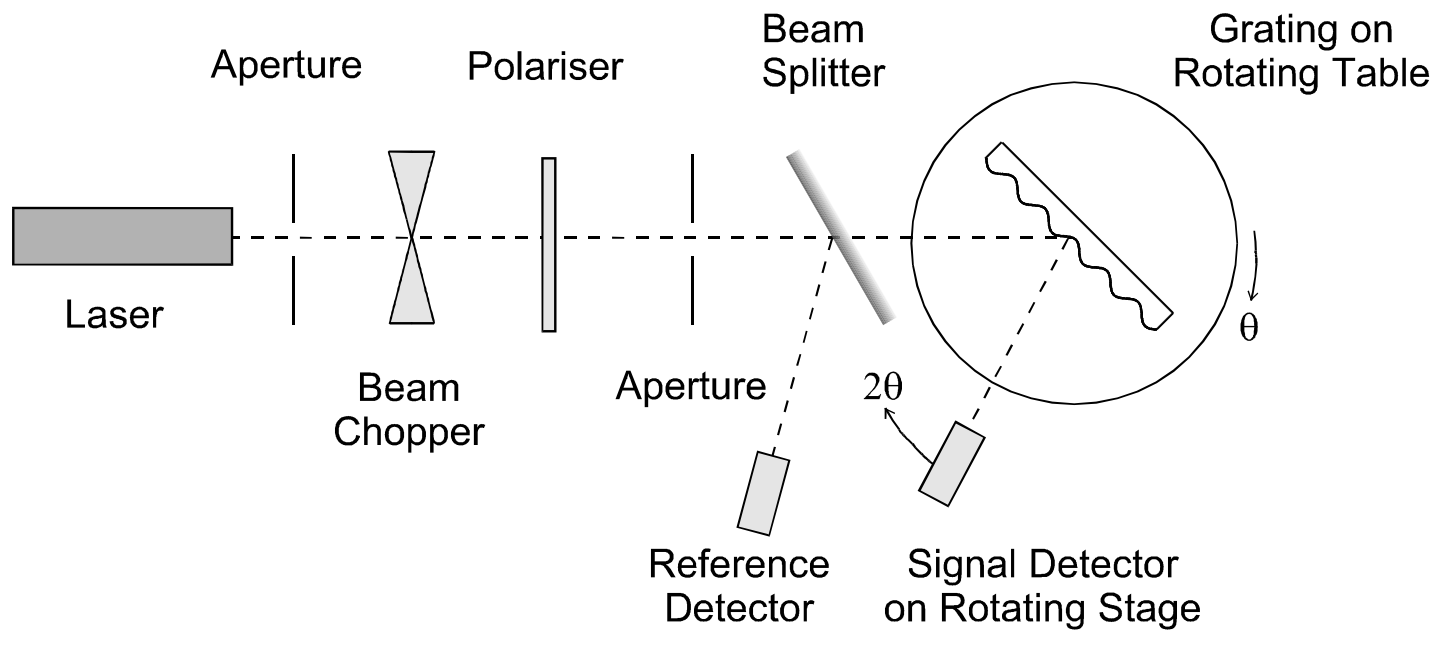




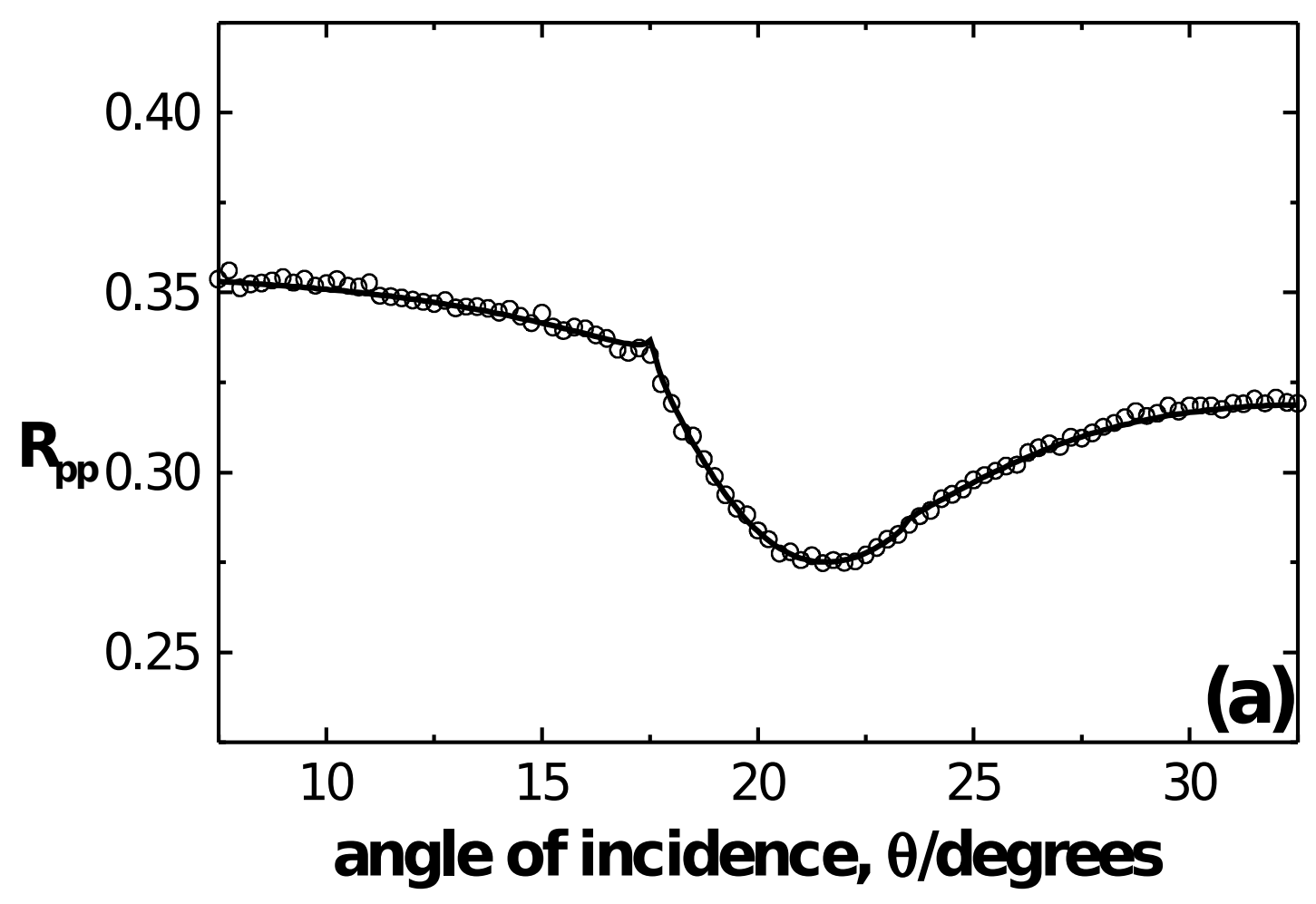



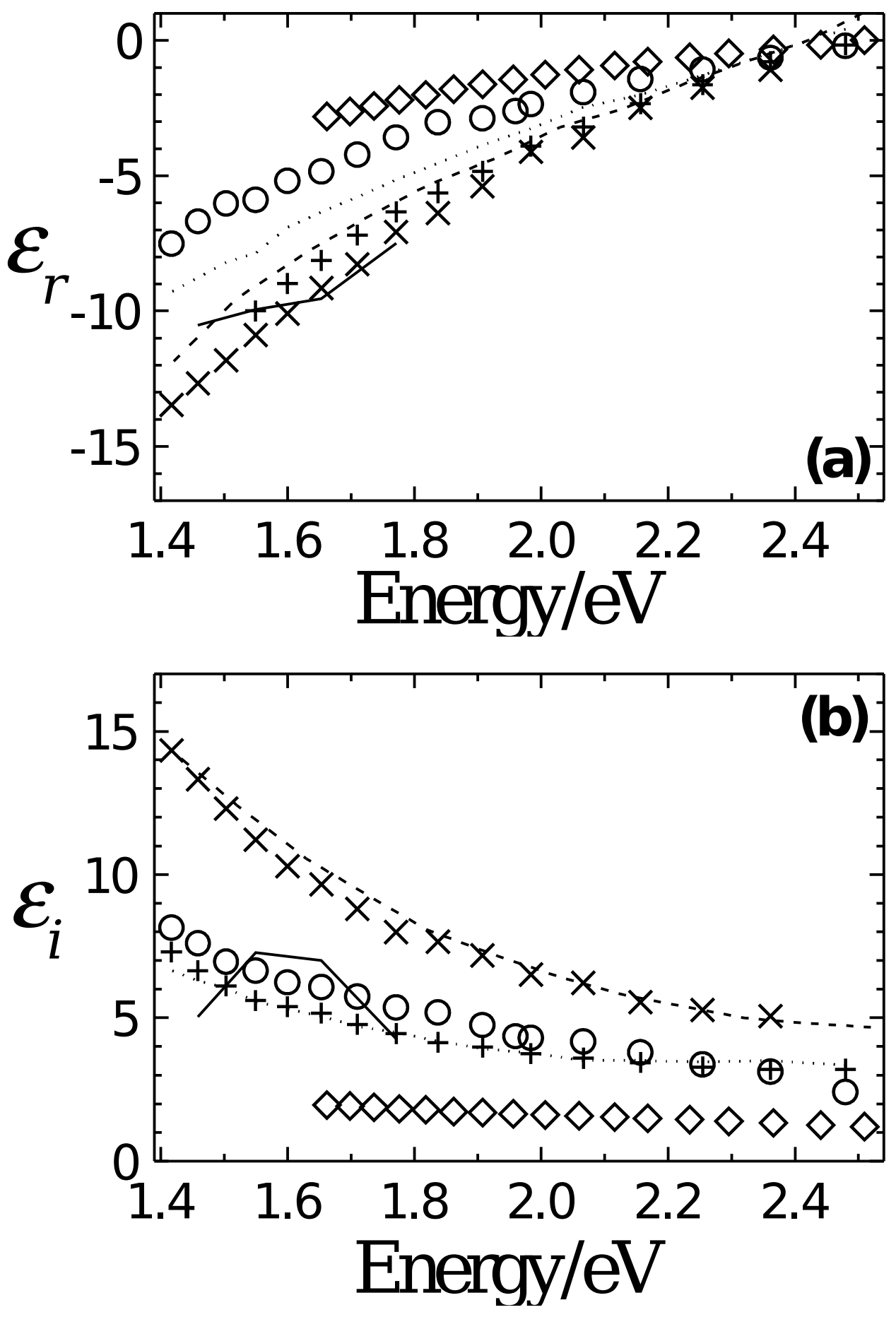

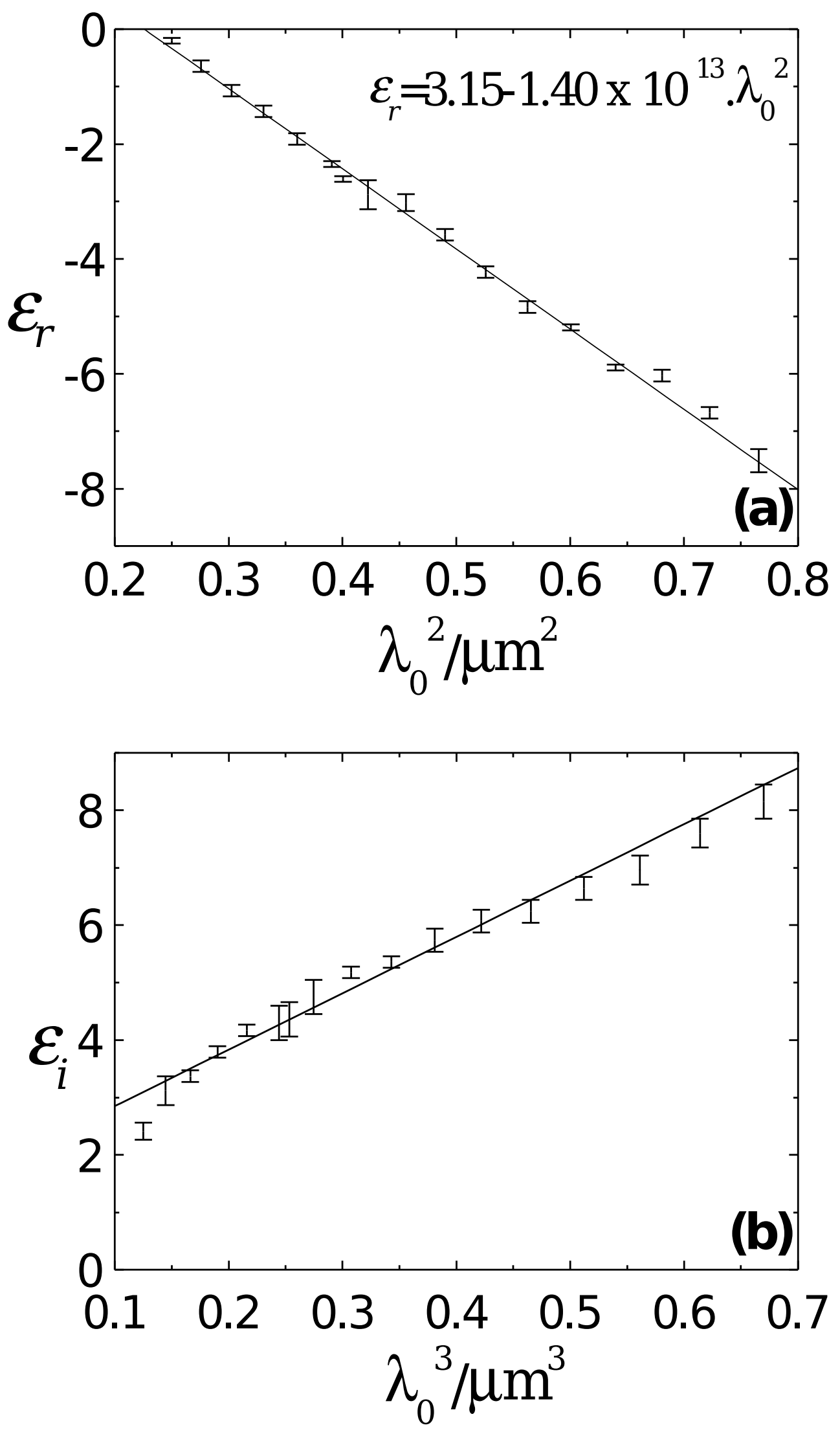


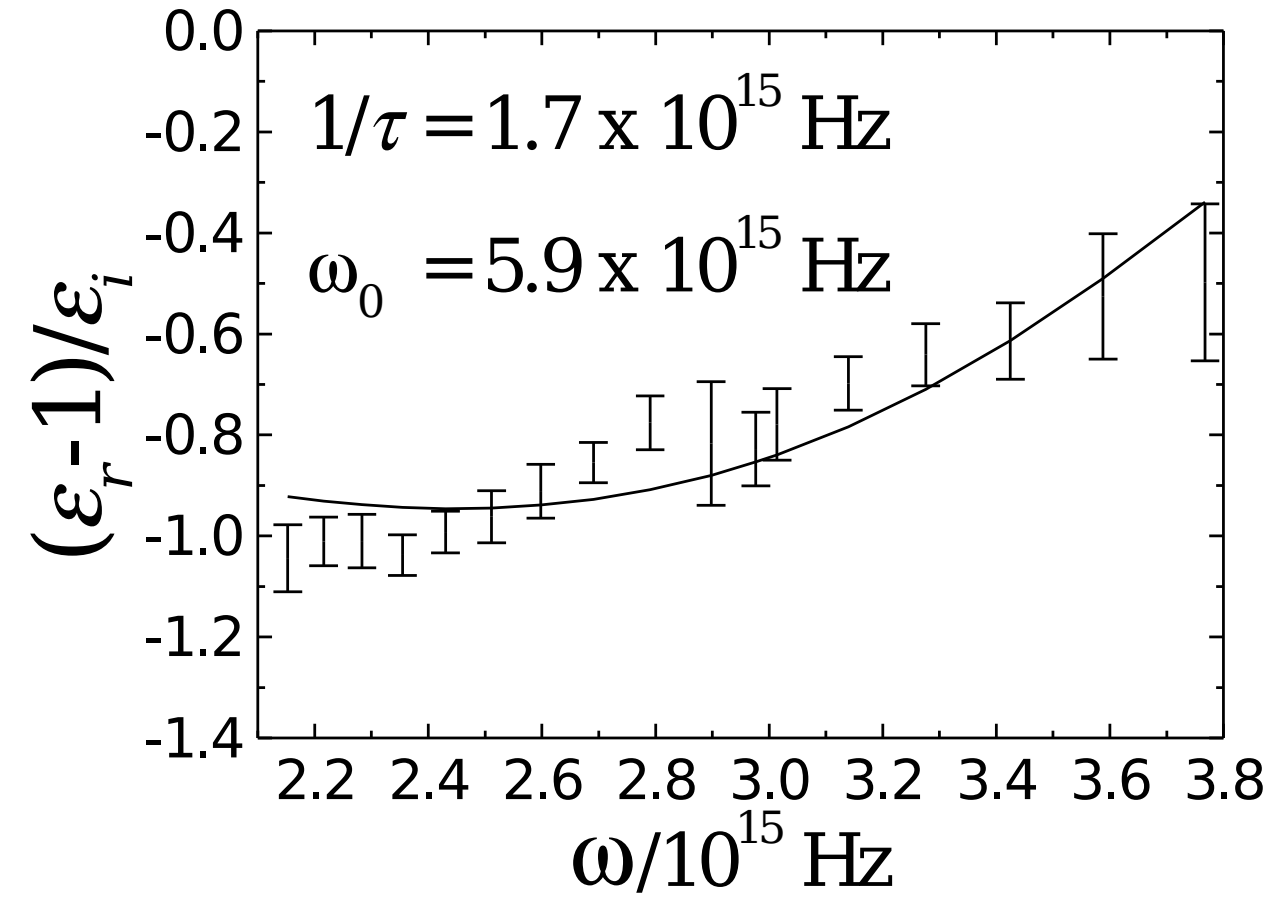



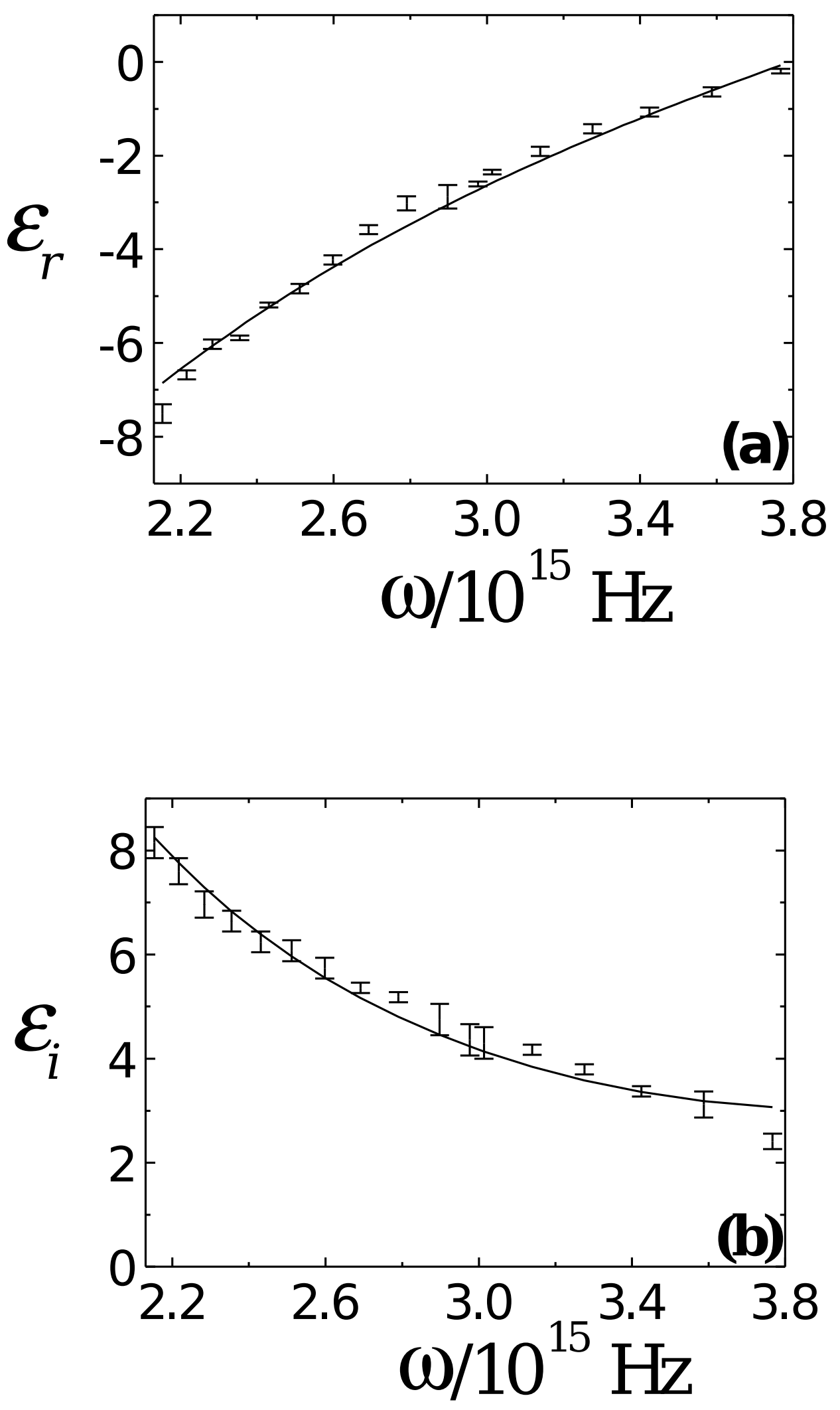